\title{
Prediagnostic serum tocopherol levels and the risk of Non- Hodgkin Lymphoma: The Multiethnic Cohort
}

\author{
Yukiko Morimoto ${ }^{1}$, Nicholas J. Ollberding ${ }^{2}$, Robert V. Cooney ${ }^{3}$, Lynne R. Wilkens ${ }^{1}$, Adrian \\ A. Franke ${ }^{1}$, Loïc Le Marchand ${ }^{1}$, Marc T. Goodman ${ }^{4}$, Brenda Y. Hernandez ${ }^{1}$, Laurence N. \\ Kolonel $^{1}$, and Gertraud Maskarinec ${ }^{1}$ \\ ${ }^{1}$ University of Hawaii Cancer Center, Honolulu, HI \\ ${ }^{2}$ Cincinnati Children's Hospital Medical Center, Cincinnati, $\mathrm{OH}$ \\ ${ }^{3}$ Department of Public Health Sciences, University of Hawaii, Honolulu, HI \\ ${ }^{4}$ Community and Population Health Research Institute, Cedars-Sinai Medical Center, Los \\ Angeles, CA
}

\section{Abstract}

Background-Compromised immunity and chronic inflammation are thought to contribute to the development of non-Hodgkin lymphoma (NHL). Because tocopherols protect cells through antioxidant mechanisms, they may play a role in NHL etiology.

\begin{abstract}
Methods-This nested case-control study within the Multiethnic Cohort examined the association of prediagnostic serum tocopherols levels measured in 271 NHL cases and 538 matched controls by high pressure liquid chromatography/photodiode-array detection with NHL risk. Conditional logistic regression was used to calculate odds ratios (ORs) and 95\% confidence intervals (CI).
\end{abstract}

Results -We observed U-shaped associations with NHL for total and a-tocopherols ( $P_{\text {trend }}<0.01$ for polynomial terms [3 df]). The ORs $(95 \% \mathrm{CI})$ for total tocopherols, which consisted primarily of a-tocopherol, were 0.41 (0.25-0.68), 0.52 (0.32-0.85), 0.39 (0.23-0.65), and $0.78(0.47-1.29)$ for the $2^{\text {nd }}-5^{\text {th }}$ quintiles as compared to the 1 st. The risk estimates were similar for $\mathrm{a}$-tocopherol but non-significant for $\beta$ - and $\gamma$-tocopherol combined and for $\delta$-tocopherol. Adjustment for serum lipids strengthened the non-linear associations for total and a-tocopherols. Serum total tocopherol levels were higher for vitamin E supplement users at cohort entry than non-users (21.32 \pm 9.04 vs $17.72 \pm 7.43 \mu \mathrm{g} / \mathrm{mL} ; P<0.0001)$, but supplement use was not associated with NHL risk. No heterogeneity in risk estimates was detected by sex, ethnicity, vitamin E supplement use, or NHL subtype.

Corresponding Author: Gertraud Maskarinec, MD, PhD, University of Hawaii Cancer Center, 701 Ilalo Street, Honolulu, HI 96813; gertraud@cc.hawaii.edu.

Disclosure of Potential Conflict of Interest: no potential conflicts of interest were disclosed.

Authors' Contributions

Conception and design: R.V. Cooney, L.R. Wilkens, A.A. Franke, L. Le Marchand, M.T. Goodman, B.Y. Hernandez, L.N. Kolonel, G. Maskarinec

Development of methodology: L.N. Kolonel, G. Maskarinec, R.V. Cooney, L.R. Wilkens

Acquisition of data: L.N. Kolonel, R.V. Cooney, L.R. Wilkens, A.A. Franke, G. Maskarinec

Analysis and interpretation of data: Y. Morimoto, N.J. Ollberding, R.V. Cooney, L.R. Wilkens, G. Maskarinec

Writing, review, and/or revision of the manuscript: Y. Morimoto, N.J. Ollberding, R.V. Cooney, L.R. Wilkens, A.A. Franke, L. Le Marchand, M.T. Goodman, B.Y. Hernandez, L.N. Kolonel, G. Maskarinec

Administrative, technical, or material support: Y. Morimoto, N.J. Ollberding, L.R. Wilkens

Study supervision: G. Maskarinec 
Conclusions-Circulating tocopherols, at levels likely reflecting adequate dietary intakes, may be protective against NHL, whereas higher intakes from supplementation may not be beneficial.

Impact-The association between serum tocopherol levels and NHL risk provides possible new insights into the etiology of NHL.

\section{Keywords}

non-Hodgkin lymphoma; tocopherols; ethnicity; prospective cohort; nested case-control study

\section{Introduction}

The tocopherols, consisting of $\alpha, \beta, \gamma$, and $\delta$ isomers, are a family of closely related molecules collectively referred to as vitamin $\mathrm{E}$, an essential nutrient in humans that must be obtained from the diet or supplementation $(1,2)$. In the United States, the recommended dietary allowance for vitamin E, estimated as $15 \mathrm{mg} /$ day for adults, is set specifically for atocopherol due to its higher vitamin E bioactivity and abundance in the circulation (1). Other tocopherols are present at lower levels in plasma and body tissues due to their lower binding constant with hepatic a-tocopherol transfer protein (3). In the contemporary American diet, $Y$-tocopherol is the most abundant form of vitamin $E$ from food sources, mainly from vegetable oils, whereas a-tocopherol is the primary form ingested via dietary supplements $(1,4,5)$. Thus, the composition of individual tocopherols in the diet is not directly reflected in blood and tissue content, which normally consists of $80 \%$ a-tocopherol (3). Since blood levels are maintained within a narrow range, $\mathbf{a}$-tocopherol levels appear to plateau during high-dose supplementation $(1,6-8)$.

Due to their antioxidant activity against a variety of oxidants generated in vivo, tocopherol isomers may protect against cancer and other diseases related to oxidative damage. In addition, individual tocopherols appear to have various anti-inflammatory, apoptosisinducing, and anti-proliferative activities $(2,9)$. Specific support for a protective role of tocopherols in NHL etiology was suggested by inverse associations between dietary consumption of a-tocopherol and NHL risk observed in previous case-control studies (1012 ), although null findings have also been reported by other case-control and prospective cohort studies (13-15). Similar equivocal findings exist for other cancers (2). Nevertheless, the existing evidence generally links antioxidant-rich plant-based food sources with a lower risk of NHL (16). Interestingly, two large-scale intervention studies showed that low-dose supplementation with a-tocopherol was protective against prostate cancer in male smokers, whereas high dose supplementation increased the risk in both smokers and non-smokers (17, 18). Also, two meta-analyses found a slight increase in all-cause mortality for vitamin $E$ intakes at supplement levels $(19,20)$ suggesting that vitamin $\mathrm{E}$ intake from dietary sources may be protective, whereas higher intakes typically obtained through supplementation may offer no benefit and could possibly be harmful (21). However, the limited evidence from the questionnaire-based dietary studies $(10,11,13,14)$ suggests no appreciable impact of supplementation on NHL risk. Given the low correlation between dietary intake and blood/ body tissue content of individual tocopherols, circulating tocopherol concentration as a biomarker may provide new insights into the possible cancer-protective role of individual tocopherols (1). Based on the reports of a non-linear relationship between tocopherol intake and cancer risk, we applied polynomial regression models to explore whether prediagnostic serum levels of tocopherols were associated with NHL risk in a nested case-control study of men and women who participated in the biospecimen subcohort of the Multiethnic Cohort (MEC) Study. 


\section{Materials and Methods}

\section{Study population}

The MEC is a longitudinal study designed to investigate the association of dietary, lifestyle, and genetic factors with cancer incidence. The cohort was assembled in Hawaii and Los Angeles in 1993-1996. Details on recruitment and baseline information have been reported previously (22). Briefly, subjects from 5 main ethnic groups (whites, Japanese Americans, Latinos, African Americans, and Native Hawaiians) were identified through drivers' license files, supplemented with voter registration lists in Hawaii and Medicare files in California. Subjects were recruited by mailing a self-administered, 26-page food frequency questionnaire (FFQ) that included information on anthropometric measures, medical history, family history of cancer, and lifestyle factors. A total of 215,251 men and women aged 45 to 75 years were included at baseline and formed a representative group of the general population as verified by a comparison of the cohort distributions with corresponding census data for the two geographical areas (22). The study protocol was approved by the Institutional Review Boards of the University of Hawaii and the University of Southern California.

The MEC biospecimen subcohort was established primarily in 2001-2006 by asking surviving cohort members to provide specimens of blood and urine (23). In total, 67,594 cohort members contributed to the prospective biorepository. When comparing the characteristics of individuals who provided specimens with those who did not, participants were older by an average of 3 years, had less education, and were more likely to be current smokers. However, there were no substantial differences for several demographic characteristics and cancer risk factors including body mass index (BMI), dietary fat and vegetable intake, physical activity, and family history of cancer suggesting that the biospecimen repository participants are broadly representative of all cohort members.

\section{Identification and selection of cases and controls}

Incident cancer cases within the MEC were identified by routine linkages to the Hawaii Tumor Registry, the Los Angeles County Cancer Surveillance Program, and the State of California Cancer Registry, all participants of the National Cancer Institute's Surveillance, Epidemiology, and End Results (SEER) program (24). NHL cases diagnosed between 1996 and 2010 were classified according to the International Classification of Diseases for Oncology, Third Edition (ICD-O-3) and aggregated into subtypes in accordance with the proposed hierarchical classification of lymphoid neoplasms for epidemiologic research (25). The subtypes included diffuse large B-cell lymphoma (DLBCL), chronic lymphocytic leukemia or small lymphocytic lymphoma (CLL/SLL), follicular lymphoma (FL), T-cell lymphomas (all types), and Other. Controls were identified from participants in the biospecimen subcohort who were alive and free of a diagnosis of NHL at the age of the case's diagnosis and matched on sex, date of birth (within \pm 1 year), ethnicity (white, Japanese American, Latino, African American, or Native Hawaiian), location (California or Hawaii), date of blood draw (within \pm 1 year), time of blood draw, and fasting hours prior to blood draw $(0-<6,6-<8,8-<10$, or $\geq 10)$. A total of 271 cases were matched to 538 controls for the present analyses ( 267 cases were matched with 2 controls, and 4 cases could only be matched with 1 control due to lack of tocopherol measures).

\section{Serum assays}

Frozen serum samples were retrieved from the MEC biorepository in matched case-control sets, thawed, and analyzed together within batches by laboratory personnel blinded to the case status of samples. Quantification of $\alpha-, \beta-, \gamma^{-}$, and $\delta$-tocopherols was performed using high pressure liquid chromatography with photodiode-array detection according to our 
earlier protocol (26-28). The $\beta$ - and $\gamma$-tocopherols were measured together. Given that $\beta$ tocopherol comprises only a tiny fraction of total tocopherols, the combined values represent predominantly $\mathrm{Y}$-tocopherols. Based on 9 triplet pooled samples prepared for quality control, the respective within- and between-batch coefficients of variation of serum tocopherols were $1.9 \pm 1.3 \%$ and $5.1 \pm 5.7 \%$ for total tocopherols; $1.8 \pm 1.3 \%$ and $5.0 \pm 5.8 \%$ for a-tocopherol; $2.2 \pm 2.0 \%$ and $5.9 \pm 4.7 \%$ for $\beta$ - and $\gamma$-tocopherols combined; and $7.3 \pm 8.9 \%$ and $13.9 \pm 8.1 \%$ for $\delta$-tocopherol.

\section{Statistical analyses}

Characteristics of NHL cases and controls were compared using chi-square tests for categorical variables and Wilcoxon rank-sum tests for continuous variables. We applied Wilcoxon rank-sum test to examine seasonal variations (Winter [October-March] vs. nonWinter [April-September] months) in serum tocopherol levels according to blood draw date. General linear models were used to evaluate the association between serum tocopherol levels and self-reported dietary tocopherol intake (log-transformed continuous), vitamin E supplement use (yes or no) at cohort entry, and dosage among vitamin E supplement users (continuous variable). To explore possible confounding for individuals in the extreme categories of serum a-tocopherol levels, we compared participants in the lowest and highest quintile to those in the middle $\left(2^{\text {nd }}-4^{\text {th }}\right)$ quintiles. Based on the previous studies linking dietary/circulating tocopherols with cytokines with inconsistent results (29), we calculated Spearman correlation coefficients between serum tocopherol and C-reactive protein (CRP) levels measured in the same blood samples (30).

Odds ratios (ORs) and 95\% confidence intervals (CIs) were estimated using conditional logistic regression with matched sets as strata relating NHL risk with quintiles of prediagnostic levels of serum tocopherols that were determined based on the exposure distribution of both cases and controls to maximize the number of cases and controls within strata. Based on the possibility of a non-linear association of serum tocopherols with NHL risk, we applied polynomial logistic regression models to calculate $P$ for the global $2 \mathrm{df}$ Wald test of quadratic and cubic terms of serum tocopherols, as well as plotted ORs and 95\% CIs calculated by restricted cubic spline conditional logistic regression (Figure 1) to visually examine the nonlinear trends. Overall trend was tested with a global $3 \mathrm{df}$ Wald test of linear, quadratic, and cubic terms. Potential confounders assessed at baseline, i.e., years of education, alcohol consumption, smoking, BMI, physical activity, and history of blood transfusion, asthma, antihistamine use, aspirin use, and acetaminophen use were examined using data collected at cohort entry, but not included in the final models, as they were not found alone, or in combination, to change the risk estimates by more than $10 \%(24,31-34)$. However, given the influence of circulating lipids on tocopherol levels and the previously reported association between total cholesterol and NHL risk (1,35), we included serum total cholesterol plus triglyceride in the models.

Sensitivity analyses were performed by excluding cases diagnosed $<1$ year after blood draw $(\mathrm{n}=53)$ to control for early clinical disease effects; current smokers at cohort entry (42 cases, 41 controls, and another 2 cases and 84 controls from their matched sets) to control for smoking; and 34 cases and 67 matched controls to control for non-fasting values. We evaluated heterogeneity in the risk estimates for overall NHL by sex, ethnicity, and vitamin E supplement use (yes or no) at cohort entry (since updated information at time of blood draw was only partially available), and NHL subtype (DLBCL, CLL/SLL, and FL). T-cell lymphomas were not included due to the small number of cases, and "Other" due to the wide range of subtypes. Heterogeneity in risk estimates by sex, ethnicity, or vitamin E supplement use was tested by a global Wald test of the cross-product terms and all polynomial (linear, quadratic and cubic) terms; likewise, heterogeneity in risk estimates across the 3 major NHL subtypes was tested by a global Wald test of all polynomial terms in an unconditional 
polytomous logistic regression accounting for the matching factors. Additionally, given the previously-suggested subtype-specific influences of dietary tocopherols $(10,11,14)$, we conducted stratified analyses for DLBCL, CLL/SLL, and FL. SAS software version 9.3 (SAS Institute, Inc., Cary, NC) was used to perform all statistical analyses with a 2-sided $P$ of $<0.05$ considered statistically significant.

\section{Results}

The mean age at blood draw was 70.0 77.4 and $70.0 \pm 7.5$ years for cases and controls, respectively (Table 1), with mean time intervals from cohort entry to blood draw of $8.0 \pm 2.7$ and $8.3 \pm 2.5$ years $(P=0.23)$, while the mean follow-up time between blood draw and NHL diagnosis was 3.1 \pm 2.3 years. Cases and controls consisted of $27 \%$ whites, $27 \%$ Japanese Americans, 23\% Latinos, 17\% African Americans, and 6\% Native Hawaiians. The cases were similar to the controls with respect to dietary intake of total and individual tocopherols, vitamin E supplement use, education level, BMI, physical activity, and alcohol consumption. There were slightly more current/past smokers (60\% vs. $54 \%)$ and, therefore, less never smokers $(40 \%$ vs. $46 \%)$ among NHL cases than controls $(P=0.09)$. Serum tocopherol concentrations, which consisted mostly of a-tocopherol, were similar for cases and controls (Table 1). No seasonal variations in circulating levels of tocopherols were detected in cases and controls.

Vitamin E supplement use in cases and controls (29\% and 28\%, respectively) was similar to that in the entire MEC population (29\%). Total tocopherol blood levels were higher for supplement users than non-users $(21.32 \pm 9.04$ vs $17.72 \pm 7.43 \mu \mathrm{g} / \mathrm{mL} ; P<0.0001)$, and the proportion of vitamin $\mathrm{E}$ supplement users increased across quintiles of serum a-tocopherol levels; the respective values for the lowest, the three middle categories combined, and the highest quintile were $12 \%, 27 \%$, and $48 \%(P<0.001)$. On the other hand, total dietary tocopherol intake at cohort entry showed no association with serum tocopherol levels before or after adjustment for vitamin E supplement use. The mean total dietary tocopherol intake for the lowest, middle, and highest quintiles were $34 \pm 21,32 \pm 16$, and $32 \pm 17 \mathrm{mg} / \mathrm{day}$, respectively. Participants with the lowest tocopherol blood levels were more likely to be male, current/past smoker, and African American or Latino, whereas the highest quintile had a higher proportion of women and Japanese Americans (data not shown). Individuals in the lowest quintile of serum a-tocopherol also reported lower education, higher BMIs, higher red meat and total fat consumption, and lower total fruit and vegetable intake than those with higher serum a-tocopherol levels. However, these factors were not related to NHL risk, nor did they substantially impact risk estimates in multivariate regression models. In addition, NHL risk was not associated with self-reported supplement use at cohort entry after adjustment for serum lipids ( $\mathrm{OR}=1.06 ; 95 \% \mathrm{CI} 0.76-1.48 ; P=0.75)$. Serum CRP levels were inversely associated with serum a-tocopherol $(r=-0.11 ; P<0.01)$, positively related to serum $\beta$ - and $\gamma$-tocopherol $(r=0.22 ; P<0.0001)$, and not associated with total or S-tocopherol.

The ORs for total tocopherols (Table 2) were 0.41 (0.25-0.68), 0.52 (0.32-0.85), 0.39 (0.23-0.65), and $0.78(0.47-1.29)$ for the $2 \mathrm{nd}$, 3rd, 4th, and 5th quintiles as compared to the 1st. The respective ORs for a-tocopherol were 0.62 (0.39-0.98), $0.46(0.28-0.76), 0.41$ $(0.24-0.68)$, and $0.83(0.50-1.36)$. As supported by significant trend tests $\left(P_{\text {trend }}<0.01\right.$ for both) for the polynomial terms ( $3 \mathrm{df}$ ), the relation was non-linear (Figure 1). We observed somewhat similar, but non-statistically significant, U-shaped relationships for $\beta-, \gamma^{-}$, and $\delta$ tocopherols. Adjustment for serum lipids resulted in higher ORs for the $5^{\text {th }}$ quintile suggesting a J-shaped association. Excluding the cases diagnosed $<1$ year after blood draw (Table 1), current smokers at cohort entry, or matched cases and controls with non-fasting 
blood samples had no material impact on the risk estimates for overall NHL (data not shown).

We detected no heterogeneity in risk estimates by sex, ethnicity, vitamin E supplement use (yes or no) at cohort entry (data not shown), or NHL subtype (Table 1). In stratified analyses by NHL subtype, total tocopherols generally showed non-linear associations with DLBCL, CLL/SLL, and FL, but none of these results reached statistical significance. The ORs for DLBCL were 0.74 (0.31-1.74), 0.59 (0.24-1.49), 0.54 (0.23-1.25), and 0.88 (0.35-2.18) for the $2 \mathrm{nd}, 3 \mathrm{rd}$, 4th, and 5 th quintiles as compared to the $1^{\text {st }}$. The respective ORs for CLL/SLL were $0.88(0.26,2.97), 0.48(0.14,1.61), 0.44(0.14,1.39)$, and $0.71(0.23,2.16)$, and those for FL were $0.21(0.06-0.75), 0.79(0.28-2.26), 0.19(0.05-0.73)$, and $0.63(0.19-2.06)$.

\section{Discussion}

To our knowledge, this is the first study to evaluate prediagnostic circulating tocopherol concentrations in relation to NHL risk. We observed non-linear associations between serum tocopherol levels and the incidence of NHL in our population-based cohort before and after adjustment for serum lipids; a-tocopherol, the major circulating tocopherol, and, therefore, also total tocopherol showed significant U-shaped relations with NHL risk, but the results for $\beta$ - and $\gamma$-combined and $\delta$-tocopherols were not statistically significant. The intermediate quintiles, representing total tocopherol concentrations of $13-23 \mu \mathrm{g} / \mathrm{mL}$ and a-tocopherol concentrations of $10-21 \mu \mathrm{g} / \mathrm{mL}$, were associated with the greatest risk reduction. It is difficult to determine the exact amount of dietary tocopherols that correspond to these serum values; however, we hypothesize that the lowest and highest quintiles likely included individuals with suboptimal dietary intake and high-dose supplement use, respectively. In fact, the proportion of self-reported vitamin E supplement users at cohort entry was higher in the highest quintile of circulating a-tocopherol levels, but we could not substantiate a lower dietary vitamin $\mathrm{E}$ intake at cohort entry for participants in the lowest quintile. Because this group differed with respect to several dietary and lifestyle risk factors, very low circulating tocopherols may also reflect poor dietary patterns and/or health behaviors that possibly influence NHL risk.

No heterogeneity in risk estimates was detected by sex, ethnicity, self-reported vitamin E supplement use, or NHL subtype in the current study, whereas previous reports have shown different patterns of association for DLBCL, CLL/SLL, and FL (10, 11, 15). Although cigarette smoking is known to modify the oxidation of circulating tocopherols, we observed no difference in risk estimates after the exclusion of cohort members who reported smoking at cohort entry (36), but the overall proportion of smokers was only <20\% at cohort entry and has decreased since then. Circulating $a$ - and $y$-tocopherol levels appear to be similar among smokers and non-smokers, probably because tocopherol concentrations in the blood are constantly repleted through a homeostatic mechanism that releases tissue reserves back into the blood $(8,36)$.

Due to the known differences between dietary intake and circulating levels of tocopherols in non-deficient individuals, the current results cannot be simply compared to past case-control and cohort studies that evaluated linear associations between dietary and/or supplement intake of tocopherols and NHL risk $(10-12,14,15,37)$. The findings from previous dietary assessment studies have been inconsistent with three case-control studies reporting inverse associations between tocopherol intakes and NHL risk (10-12), whereas a case-control study and two cohort studies reported no association for dietary and supplement intake (1315). Conflicting findings in dietary studies of NHL and other cancers may be partly due to limitations of FFQs to quantify the type and frequency of different vegetable oils used and, hence, to estimate dietary tocopherol intake. Another research constraint is the weak 
correlations of dietary and supplement intake with relative blood and tissue compositions of this nutrient in the absence of deficiency (2). The lack of strong linear associations reported in past studies (38-40) comparing dietary intake using FFQ, diet history, or diet record and blood levels of a-tocopherol portraits the inadequacy of self-reported dietary intake in providing an accurate measure of an individual's vitamin E status.

Our current finding of a U-shaped association agrees with a prostate cancer study in smokers describing non-linear associations of baseline serum tocopherol levels with all-cause and cancer-specific mortality after 16 years of follow-up (7). The maximal risk reduction in that study was observed at $13-14 \mu \mathrm{g} / \mathrm{mL}$, while the optimal range in our study was estimated at $10-21 \mu \mathrm{g} / \mathrm{mL}$ suggesting a possible benefit of low to moderate supplementation. This (7) and another prospective study (41) also reported inverse associations of prediagnostic serum a-tocopherol levels with prostate cancer risk, and the associations were stronger in supplement users and smokers. In addition, daily treatment with a high dose a-tocopherol supplement (400 IU) suppressed blood $\mathrm{Y}$-tocopherol levels throughout the intervention period of 4 years or more (7) suggesting that high intakes of a-tocopherol lower the blood levels of other tocopherols $(2,21)$. Since all tocopherols possess diverse and unique functions (21), an excessive intake of a-tocopherol may suppress the beneficial actions exerted by other tocopherols present in low concentrations, while low-dose supplementation may avoid these suppressive effects and be beneficial in populations such as smokers who experience higher oxidative stress. The inverse association between serum a-tocopherol and CRP may suggest a possible mechanism of action through an anti-inflammatory pathway in the etiology of NHL (29). In contrast to a-tocopherol, $\mathrm{Y}$ - tocopherol has anti-inflammatory properties and circulating levels appear to rise in response to inflammation, thereby complicating the interpretation of the observed associations.

This study had several important strengths. This was the first study to evaluate serum total and individual tocopherol levels prior to NHL diagnosis in a population-based prospective study design, and the cases and controls consisted of supplement vitamin E users and nonusers. The total sample size was relatively large for a cancer with low incidence and included multiple ethnic groups that likely had more diverse patterns of dietary consumption and supplement use than a more homogeneous population of a single ethnic origin, resulting in a wider range of vitamin E intakes. The NHL diagnosis was confirmed by SEER cancer registries with excellent quality control, and data related to other potential risk factors were also available. The interpretation of the results, however, needs caution given the multiple subtypes with etiologic diversity (25). The values derived from prediagnostic specimens are not subject to the recall biases or measurement error inherent with FFQ-based data from case-control studies (42). Even in prospective studies, intake of tocopherols is difficult to estimate because, as mentioned earlier, it requires detailed quantification of the intake of different added vegetable oils (1). Therefore, it was not surprising to find weak correlations between FFQ-based dietary intake and serum values of tocopherols. In addition, the time gap of nearly a decade between the baseline FFQ and blood draw likely contributed to the lack of correlation. Thus, the evaluation of circulating tocopherol concentrations offers a more direct measure of antioxidant bioactivity than dietary intake.

The current investigation also had a number of limitations. Most importantly, the mean follow-up time of 3.1 years between blood collection and NHL diagnosis was short. With little knowledge about the latency period between dietary exposure and the development of NHL, we cannot rule out the possibility that our results, in part, reflect underlying physiological changes resulting from subclinical disease present prior to diagnosis, although excluding the first year of follow-up had no impact on the findings. Although the use of a single measurement may have misclassified exposure status for some individuals and, thereby, attenuated risk estimates, serum tocopherol levels measured over time show greater 
stability than other biomarkers with correlations of $>0.7$ over $2-4$ weeks (43). Also, we did not have updated information on smoking status, dietary intake, or supplement use at the time of blood draw for all cases and controls. Lastly, we cannot rule out residual confounding, and the small sample size did not allow for robust risk estimates and detailed analyses by NHL subtype or smoking status. Nevertheless, our preliminary results showing associations despite limited statistical power provide the basis for further investigations into a possible link between circulating tocopherols and NHL risk.

In conclusion, our data provide support for a protective role of circulating tocopherols at levels reflecting adequate dietary intakes in NHL development. Although our results need to be confirmed in future studies with longer follow-up, larger samples to allow for analysis of NHL subtypes and, ideally, repeated tocopherol measurements over time, the U-shaped associations support the emerging hypothesis that intakes from high-dose supplementation are not beneficial. A better understanding of the cancer-protective functions of individual tocopherol isomers is needed to interpret the observed non-linear associations, if confirmed, and to determine the optimal concentrations of these isomers in blood and tissue with the corresponding dietary and/or supplement requirements.

\section{Acknowledgments}

The authors thank all participants in the Multiethnic Cohort Study and Cynthia Morrison for the technical performance of the HPLC and autoanalyzer assays.

Financial Support: the Multiethnic Cohort Study was supported by National Cancer Institute grants R37 CA 54281 and P01 CA 33619 (PI: L Kolonel) and U01 CA 63464 (PI: B Henderson). NJO was supported by a postdoctoral fellowship on grant R25 CA 90956. The tumor registries in Hawaii and Los Angeles are supported by NCI contracts N01 PC 35137 and N01 PC 35139. Performance of the serum tocopherol assays was supported in part by NCI award P30 CA71789.

\section{References}

1. Institute of Medicine FaNB. Dietary Reference Intakes for Vitamin C, Vitamin E, Selenium, and Carotenoids. National Academy Press; Washington, DC: 2000. http://www.nap.edu/catalog.php? record_id=9810\#toc

2. Ju J, Picinich SC, Yang Z, Zhao Y, Suh N, Kong AN, et al. Cancer-preventive activities of tocopherols and tocotrienols. Carcinogenesis. 2010; 31:533-42. [PubMed: 19748925]

3. Herrera E, Barbas C. Vitamin E: action, metabolism and perspectives. J Physiol Biochem. 2001; $57: 43-56$.

4. Office of Dietary Supplements NIoH. [Accessed on 1-31-2013.] Dietary supplement fact sheet: Vitamin E. 10-11-2011. ods.od.nih.gov/factsheets/VitaminE-HealthProfessional/

5. Dietrich M, Traber MG, Jacques PF, Cross CE, Hu Y, Block G. Does gamma-tocopherol play a role in the primary prevention of heart disease and cancer? A review. J Am Coll Nutr. 2006; 25:292-9. [PubMed: 16943450]

6. Dimitrov NV, Meyer C, Gilliland D, Ruppenthal M, Chenoweth W, Malone W. Plasma tocopherol concentrations in response to supplemental vitamin E. Am J Clin Nutr. 1991; 53:723-9. [PubMed: 2000827]

7. Lippman SM, Klein EA, Goodman PJ, Lucia MS, Thompson IM, Ford LG, et al. Effect of selenium and vitamin E on risk of prostate cancer and other cancers: the Selenium and Vitamin E Cancer Prevention Trial (SELECT). JAMA. 2009; 301:39-51. [PubMed: 19066370]

8. Brigelius-Flohe R, Traber MG. Vitamin E: function and metabolism. FASEB J. 1999; 13:1145-55. [PubMed: 10385606]

9. Constantinou C, Papas A, Constantinou AI. Vitamin E and cancer: An insight into the anticancer activities of vitamin E isomers and analogs. Int J Cancer. 2008; 123:739-52. [PubMed: 18512238]

10. Chang ET, Balter KM, Torrang A, Smedby KE, Melbye M, Sundstrom C, et al. Nutrient intake and risk of non-Hodgkin's lymphoma. Am J Epidemiol. 2006; 164:1222-32. [PubMed: 17005624] 
11. Holtan SG, O’Connor HM, Fredericksen ZS, Liebow M, Thompson CA, Macon WR, et al. Foodfrequency questionnaire-based estimates of total antioxidant capacity and risk of non-Hodgkin lymphoma. Int J Cancer. 2012; 131:1158-68. [PubMed: 22038870]

12. Polesel J, Talamini R, Montella M, Parpinel M, Dal ML, Crispo A, et al. Linoleic acid, vitamin D and other nutrient intakes in the risk of non-Hodgkin lymphoma: an Italian case-control study. Ann Oncol. 2006; 17:713-8. [PubMed: 16556850]

13. Kelemen LE, Cerhan JR, Lim U, Davis S, Cozen W, Schenk M, et al. Vegetables, fruit, and antioxidant-related nutrients and risk of non-Hodgkin lymphoma: a National Cancer InstituteSurveillance, Epidemiology, and End Results population-based case-control study. Am J Clin Nutr. 2006; 83:1401-10. [PubMed: 16762953]

14. Zhang SM, Hunter DJ, Rosner BA, Giovannucci EL, Colditz GA, Speizer FE, et al. Intakes of fruits, vegetables, and related nutrients and the risk of non-Hodgkin's lymphoma among women. Cancer Epidemiol Biomarkers Prev. 2000; 9:477-85. [PubMed: 10815692]

15. Thompson CA, Habermann TM, Wang AH, Vierkant RA, Folsom AR, Ross JA, et al. Antioxidant intake from fruits, vegetables and other sources and risk of non-Hodgkin's lymphoma: the Iowa Women's Health Study. Int J Cancer. 2010; 126:992-1003. [PubMed: 19685491]

16. Chen GC, Lv DB, Pang Z, Liu QF. Fruits and vegetables consumption and risk of non-Hodgkin's lymphoma: a meta-analysis of observational studies. Int J Cancer. 2013; 133:190-200. [PubMed: 23238796]

17. Klein EA, Thompson IM Jr, Tangen CM, Crowley JJ, Lucia MS, Goodman PJ, et al. Vitamin E and the risk of prostate cancer: the Selenium and Vitamin E Cancer Prevention Trial (SELECT). JAMA. 2011; 306:1549-56. [PubMed: 21990298]

18. Heinonen OP, Albanes D, Virtamo J, Taylor PR, Huttunen JK, Hartman AM, et al. Prostate cancer and supplementation with alpha-tocopherol and beta-carotene: incidence and mortality in a controlled trial. J Natl Cancer Inst. 1998; 90:440-6. [PubMed: 9521168]

19. Miller ER III, Pastor-Barriuso R, Dalal D, Riemersma RA, Appel LJ, Guallar E. Meta-analysis: high-dosage vitamin E supplementation may increase all-cause mortality. Ann Intern Med. 2005; 142:37-46. [PubMed: 15537682]

20. Bjelakovic G, Nikolova D, Gluud LL, Simonetti RG, Gluud C. Antioxidant supplements for prevention of mortality in healthy participants and patients with various diseases. Cochrane Database Syst Rev. 2012; 3:CD007176. [PubMed: 22419320]

21. Yang CS, Suh N, Kong AN. Does vitamin E prevent or promote cancer? Cancer Prev Res (Phila). 2012; 5:701-5. [PubMed: 22490437]

22. Kolonel LN, Henderson BE, Hankin JH, Nomura AMY, Wilkens LR, Pike MC, et al. A multiethnic cohort in Hawaii and Los Angeles: baseline characteristics. Am J Epidemiol. 2000; 151:346-57. [PubMed: 10695593]

23. Park SY, Wilkens LR, Henning SM, Le ML, Gao K, Goodman MT, et al. Circulating fatty acids and prostate cancer risk in a nested case-control study: the Multiethnic Cohort. Cancer Causes Control. 2009; 20:211-23. [PubMed: 18821021]

24. Maskarinec G, Erber E, Gill J, Cozen W, Kolonel LN. Overweight and obesity at different times in life as risk factors for non-Hodgkin's lymphoma: the multiethnic cohort. Cancer Epidemiol Biomarkers Prev. 2008; 17:196-203. [PubMed: 18187389]

25. Turner JJ, Morton LM, Linet MS, Clarke CA, Kadin ME, Vajdic CM, et al. InterLymph hierarchical classification of lymphoid neoplasms for epidemiologic research based on the WHO classification (2008): update and future directions. Blood. 2010; 116:e90-e98. [PubMed: 20699439]

26. Franke AA, Custer LJ, Cooney RV. Synthetic carotenoids as internal standards for plasma micronutrient analyses by high-performance liquid chromatography. J Chromatogr. 1993; 614:4357. [PubMed: 8496285]

27. Cooney RV, Franke AA, Hankin JH, Custer LJ, Wilkens LR, Harwood PJ, et al. Seasonal variations in plasma micronutrients and antioxidants. Cancer Epidemiol Biomarkers Prev. 1995; 4:207-15. [PubMed: 7606195] 
28. Epplein M, Franke AA, Cooney RV, Morris JS, Wilkens LR, Goodman MT, et al. Association of plasma micronutrient levels and urinary isoprostane with risk of lung cancer: the multiethnic cohort study. Cancer Epidemiol Biomarkers Prev. 2009; 18:1962-70. [PubMed: 19531680]

29. Calder PC, Ahluwalia N, Brouns F, Buetler T, Clement K, Cunningham K, et al. Dietary factors and low-grade inflammation in relation to overweight and obesity. Br J Nutr. 2011; 106 (Suppl 3):S5-78. [PubMed: 22133051]

30. Conroy SM, Maskarinec G, Morimoto Y, Franke AA, Cooney RV, Wilkens LR, et al. NonHodgkin Lymphoma and circulating markers of inflammation and adiposity in a nested casecontrol study: The Multiethnic Cohort. Cancer Epidemiol Biomarkers Prev. 2013

31. Skibola CF. Obesity, diet and risk of non-Hodgkin lymphoma. Cancer Epidemiol Biomarkers Prev. 2007; 16:392-5. [PubMed: 17337642]

32. Cross AJ, Lim U. The role of dietary factors in the epidemiology of non-Hodgkin's lymphoma. Leuk Lymphoma. 2006; 47:2477-87. [PubMed: 17169793]

33. Fisher SG, Fisher RI. The epidemiology of non-Hodgkin's lymphoma. Oncogene. 2004; 23:652434. [PubMed: 15322522]

34. Mickey RM, Greenland S. The impact of confounder selection criteria on effect estimation. Am J Epidemiol. 1989; 129:125-37. [PubMed: 2910056]

35. Morimoto Y, Conroy SM, Ollberding NJ, Henning SM, Franke AA, Wilkens LR, et al. Erythrocyte membrane fatty acid composition, serum lipids, and non-Hodgkin's lymphoma risk in a nested case-control study: the multiethnic cohort. Cancer Causes Control. 2012; 23:1693-703. [PubMed: 22907421]

36. Bruno RS, Traber MG. Vitamin E biokinetics, oxidative stress and cigarette smoking. Pathophysiology. 2006; 13:143-9. [PubMed: 16814530]

37. Kelemen LE, Wang SS, Lim U, Cozen W, Schenk M, Hartge P, et al. Vegetables- and antioxidantrelated nutrients, genetic susceptibility, and non-Hodgkin lymphoma risk. Cancer Causes Control. 2008; 19:491-503. [PubMed: 18204928]

38. Brunner E, Stallone D, Juneja M, Bingham S, Marmot M. Dietary assessment in Whitehall II: comparison of $7 \mathrm{~d}$ diet diary and food-frequency questionnaire and validity against biomarkers. $\mathrm{Br}$ J Nutr. 2001; 86:405-14. [PubMed: 11570993]

39. Stryker WS, Kaplan LA, Stein EA, Stampfer MJ, Sober A, Willett WC. The relation of diet, cigarette smoking, and alcohol consumption to plasma beta-carotene and alpha-tocopherol levels. Am J Epidemiol. 1988; 127:283-96. [PubMed: 3257350]

40. Wallstrom P, Wirfalt E, Lahmann PH, Gullberg B, Janzon L, Berglund G. Serum concentrations of beta-carotene and alpha-tocopherol are associated with diet, smoking, and general and central adiposity. Am J Clin Nutr. 2001; 73:777-85. [PubMed: 11273853]

41. Weinstein SJ, Peters U, Ahn J, Friesen MD, Riboli E, Hayes RB, et al. Serum alpha-tocopherol and gamma-tocopherol concentrations and prostate cancer risk in the PLCO Screening Trial: a nested case-control study. PLoS One. 2012; 7:e40204. [PubMed: 22792240]

42. Arab L, Akbar J. Biomarkers and the measurement of fatty acids. Public Health Nutr. 2002; 5:86571. [PubMed: 12638594]

43. Block G, Dietrich M, Norkus E, Jensen C, Benowitz NL, Morrow JD, et al. Intraindividual variability of plasma antioxidants, markers of oxidative stress, C-reactive protein, cotinine, and other biomarkers. Epidemiology. 2006; 17:404-12. [PubMed: 16755268] 


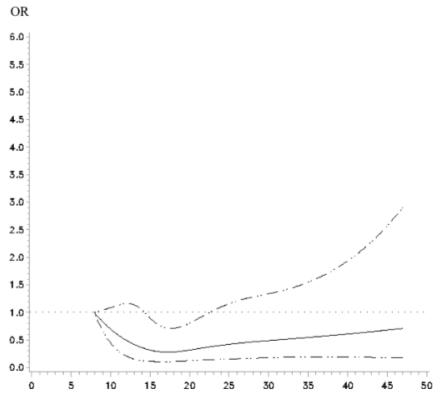

Total tocopherols $(\mu \mathrm{g} / \mathrm{mL})$

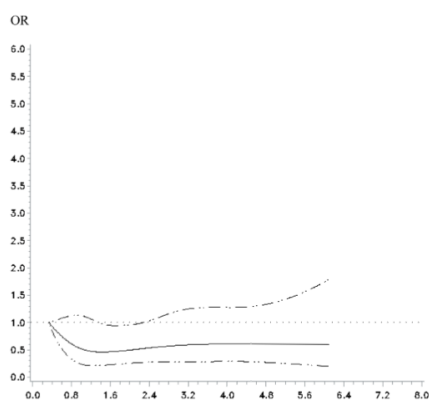

$\beta$ - and $\gamma$ - Tocopherols $(\mu \mathrm{g} / \mathrm{mL})$

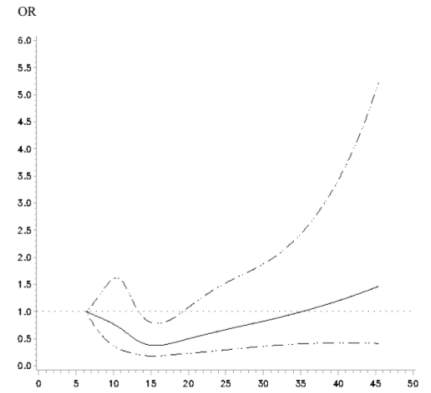

$\alpha$-Tocopherol ( $\mu \mathrm{g} / \mathrm{mL})$

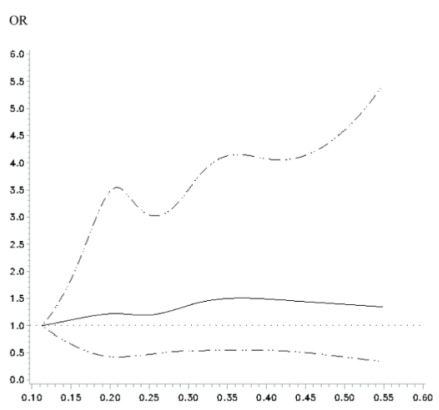

$\delta$-Tocopherol ( $\mu \mathrm{g} / \mathrm{mL})$

Figure 1. Prediagnostic serum tocopherol levels and NHL risk

Odds ratios (OR) were calculated by restricted cubic spline conditional logistic regression. Cases and controls were matched on sex, date of birth (within \pm 1 year), ethnicity (White, Japanese American, Latino, African American, or Native Hawaiian), location (California or Hawaii), date of blood draw (within \pm 1 year), time of blood draw, and fasting hours prior to blood draw $(0-<6,6-<8,8-<10$, or $\geq 10$ hours). The solid lines represent point estimates and the dashed lines $95 \%$ confidence intervals. 
Table 1

Characteristics of participants in the NHL nested case-control study *

\begin{tabular}{|c|c|c|c|}
\hline \multirow{2}{*}{ Characteristic } & \multicolumn{2}{|c|}{ Total $(\mathrm{N}=809)$} & \multirow{2}{*}{$P$ value $^{t}$} \\
\hline & Cases $(N=271)$ & Controls $(N=538)$ & \\
\hline Age at blood draw (years) $t$ & $70.0 \pm 7.4$ & $70.0 \pm 7.5$ & -- \\
\hline Sex Male & $157(58 \%)$ & $312(58 \%)$ & -- \\
\hline Female & $114(42 \%)$ & $226(42 \%)$ & \\
\hline \multicolumn{4}{|l|}{ Ethnicity $t$} \\
\hline White & $74(27 \%)$ & $148(28 \%)$ & \\
\hline Japanese American & $72(27 \%)$ & $142(26 \%)$ & \\
\hline Latino & $62(23 \%)$ & $123(23 \%)$ & \\
\hline African American & $46(17 \%)$ & $92(17 \%)$ & -- \\
\hline Native Hawaiian & $17(6 \%)$ & $33(6 \%)$ & \\
\hline Time from blood draw to NHL diagnosis (years) & $3.1 \pm 2.3$ & -- & \\
\hline Time from cohort entry to blood draw & $8.0 \pm 2.7$ & $8.3 \pm 2.5$ & 0.23 \\
\hline Hours fasting prior to blood draw $t$ & $12.0 \pm 4.0$ & $12.3 \pm 4.1$ & -- \\
\hline Blood sample collected in winter months (October-March) & $136(50 \%)$ & $242(45 \%)$ & 0.16 \\
\hline Education (years) & $13.7 \pm 3.0$ & $13.7 \pm 3.2$ & 0.94 \\
\hline Body mass index at cohort entry $\left(\mathrm{kg} / \mathrm{m}^{2}\right)$ & $26.9 \pm 5.3$ & $26.4 \pm 4.3$ & 0.47 \\
\hline \multicolumn{4}{|l|}{ Smoking status at cohort entry } \\
\hline Current/past & $163(60 \%)$ & $290(54 \%)$ & 0.09 \\
\hline Never & $108(40 \%)$ & $248(46 \%)$ & \\
\hline Physical activity at cohort entry (metabolic equivalents/day) & $1.6 \pm 0.3$ & $1.6 \pm 0.3$ & 0.10 \\
\hline Total dietary tocopherol intake at cohort entry (mg/day) & $28.5(19.4-39.3)$ & $28.7(21.0-38.8)$ & 0.81 \\
\hline a-Tocopherol & $8.0(5.8-12.0)$ & $8.5(6.1-12.1)$ & 0.35 \\
\hline$\beta$ - and $y$-Tocopherols & $15.5(10.4-22.1)$ & $15.3(10.7-20.5)$ & 0.83 \\
\hline$\delta$-Tocopherol & $4.7(3.0-6.4)$ & $4.5(3.1-6.1)$ & 0.68 \\
\hline Vitamin E supplement use at cohort entry & $79(29 \%)$ & $149(28 \%)$ & 0.66 \\
\hline Alcohol intake at cohort entry (g/day) & $9.7 \pm 24.4$ & $11.2 \pm 28.4$ & 0.71 \\
\hline \multicolumn{4}{|l|}{ Non-Hodgkin's lymphoma subtype $\mathcal{\xi}$} \\
\hline DLBCL & $78(29 \%)$ & -- & -- \\
\hline CLL/SLL & $51(19 \%)$ & -- & \\
\hline Follicular lymphoma & $49(18 \%)$ & -- & \\
\hline T-cell lymphomas & $15(5 \%)$ & -- & \\
\hline Other & $78(29 \%)$ & -- & \\
\hline Total serum tocopherols $(\mu \mathrm{g} / \mathrm{mL})$ & $16.28(12.87-22.73)$ & $16.73(13.85-21.12)$ & 0.52 \\
\hline a-Tocopherol & $13.65(10.33-20.47)$ & $14.46(11.39-19.42)$ & 0.32 \\
\hline$\beta$ - and $\gamma$-Tocopherol & $1.71(0.90-2.79)$ & $1.69(0.98-2.72)$ & 0.79 \\
\hline$\delta$-Tocopherol & $0.26(0.20-0.31)$ & $0.24(0.20-0.31)$ & 0.30 \\
\hline
\end{tabular}

Cancer Epidemiol Biomarkers Prev. Author manuscript; available in PMC 2014 November 01. 
${ }^{\dagger}$ Univariate comparisons of cases and controls were performed using chi-square test (categorical variables) and Wilcoxon rank-sum test (continuous variables).

${ }^{*}$ Characteristics included in case-control matching criteria; date of birth and date of blood draw were matched within \pm 1 year; fasting hours prior to blood draw was matched as $<6,6$ to $<8,8$ to $<10, \geq 10$ hours.

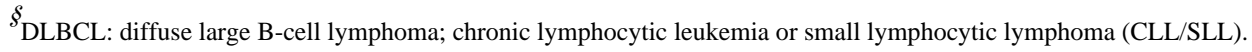




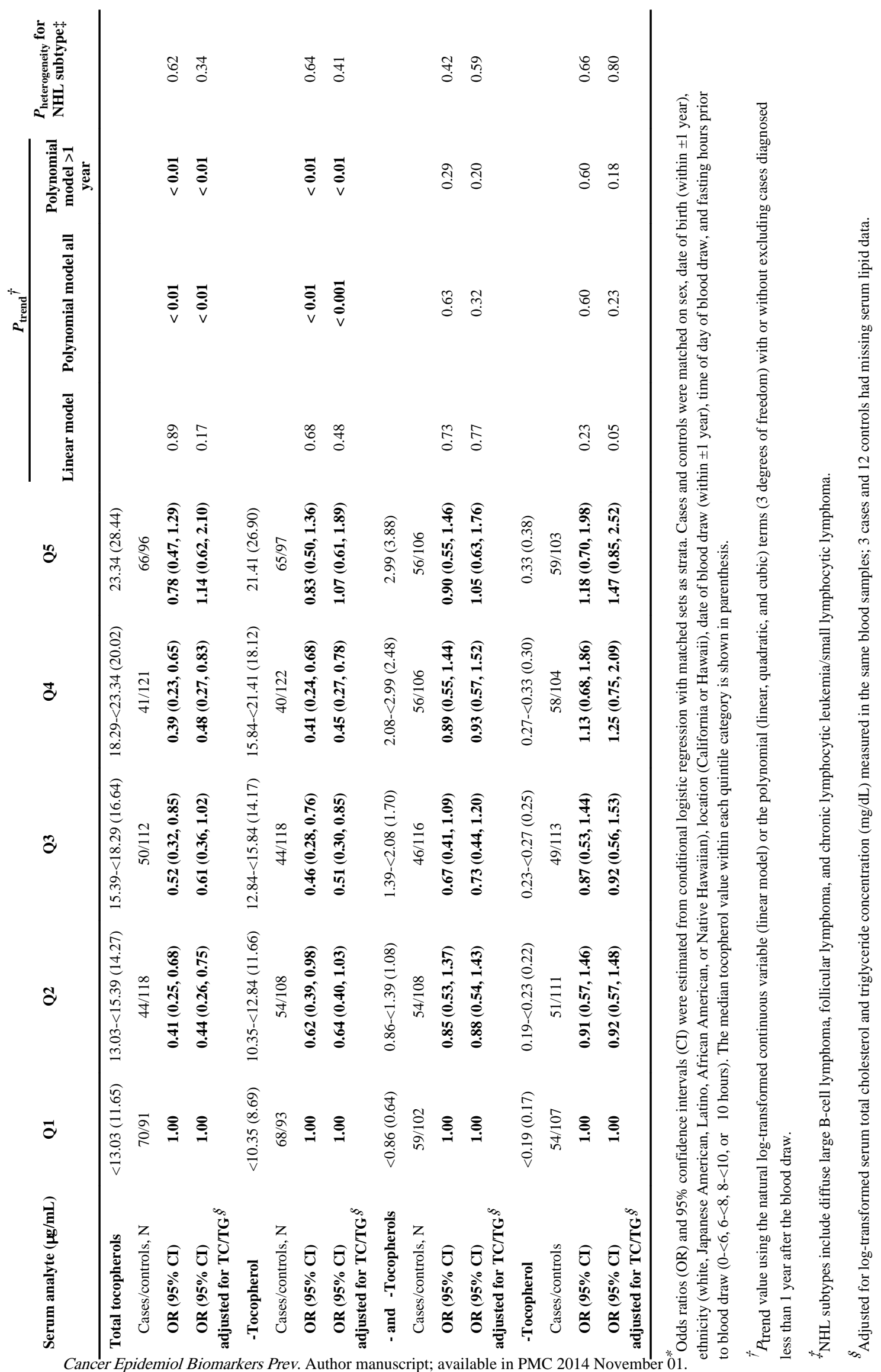

